Title : will be set by the publisher

Editors : will be set by the publisher

EAS Publications Series, Vol. ?, 2005

\title{
DOME C AS A SETTING FOR THE PERMANENT ALL SKY SURVEY (PASS)
}

\author{
H.J. Deeg, J.A. Belmonte, R. Alonso ${ }^{1}$, K. Horne, K. Alsubai ${ }^{2}$ and L.R. \\ Doyle $^{3}$
}

\begin{abstract}
The Permanent All Sky Survey (PASS) is a project for a continuous photometric survey of the entire celestial sphere with a high temporal resolution. Its major objectives are the detection of all giantplanet transits (with periods up to some weeks) across stars up to mag 10.5 , and the delivery of continuous time series photometry that is useful for the study of any variable stars. For a southern instrument of PASS, Dome C offers two major advantages over mid-latitude sites: lower noises and greatly improved observational coverage. The simplicity of the instrument should make it fairly easily adaptable to the extreme low temperatures at that site.
\end{abstract}

\section{Introduction}

The Permanent All Sky Survey (PASS) is a project for a photometric survey of the entire celestial sphere with a high temporal resolution. Its major objectives are the detection of all giant-planet transits (with periods up to some weeks) across stars up to mag 10.5, and the acquisition of photometry that is useful for the study of any variable stars, reaching to somewhat fainter magnitudes (depending on the precision required). About 250000 stars are expected to be surveyed by PASS, with the goal to obtain their light-curves with the upmost continuity on time-scales of minutes, and to link these curves into the databases that are commonly used by the astronomical community. The detection of a complete sample of about 30 transiting giant planets around all bright stars may be expected. The basic instrument design consists of several arrays of about 15 fixed CCD cameras each (Fig. 1), which will simultaneously survey the entire sky. These cameras have short (about $50 \mathrm{~mm}$ ) focal lengths, with overlapping fields of view (about $30^{\circ} \mathrm{x}$

\footnotetext{
${ }^{1}$ Instituto de Astrofísica de Canarias, C. Via Lactea S/N, 38200 La Laguna, Spain

2 School of Physics and Astronomy, University of St. Andrews, KY169SS, Scotland, UK

${ }^{3}$ SETI Institute, 515 N. Whisman Ave, Mountain View, CA 94043, USA
} 


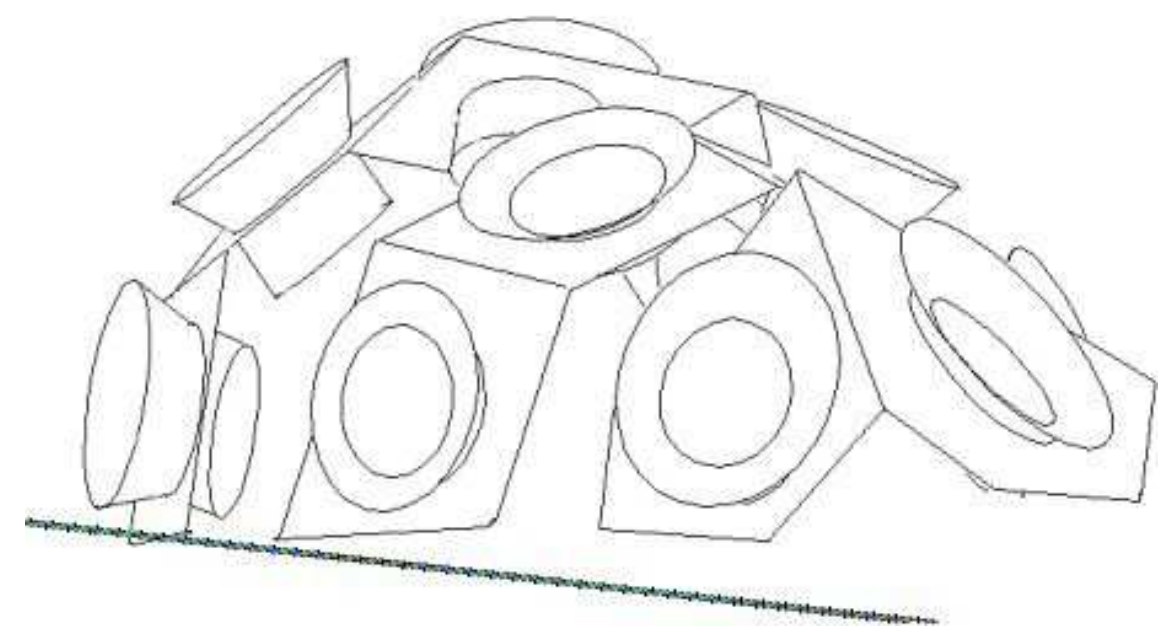

Fig. 1. Schematic design of a PASS camera array. It consists of a number of wide-field cameras which together cover the entire visible sky above a given observing location. At Dome $\mathrm{C}$, these should be enclosed into a common insulating cover with optical windows in front of each camera.

$30^{\circ}$ each), which allows a full coverage of the visible sky above $\approx 30^{\circ}$ elevation at each observing location. The cameras will continuously take short exposures (15 sec to 1 minute) in which the stars will appear as short traces. To obtain a full sky coverage, at least one array needs to be set up in both the northern and the southern hemispheres. In order to obtain a good temporal coverage, the placement of several instruments at sites distributed in longitudes is however desirable as well. In this contribution, we make the case for the placement of such an instrument at Dome C. A more detailed presentation of the objectives and design of PASS is given in Deeg et al. (2004a). A prototype of the instrument, which is currently being set up at Teide Observatory (Tenerife, Canary Islands), is described in Deeg et al. (2004b).

\section{Placement of PASS at Dome C}

For a southern instrument of PASS, a placement at Dome C may be an interesting alternative against a location at a good conventional observing site. This results from two major advantages of the Dome $\mathrm{C}$ site: lower noises and better observational coverage. About the first factor, Fig. (2a) shows the major noises affecting photometry for a normal observing site at $2400 \mathrm{~m}$ (described in detail in Deeg et al. 2004a); Fig. (2b) gives those that may be expected at Dome C. In both cases, scintillation dominates for bright stars, and the photon noise from the sky 
a)

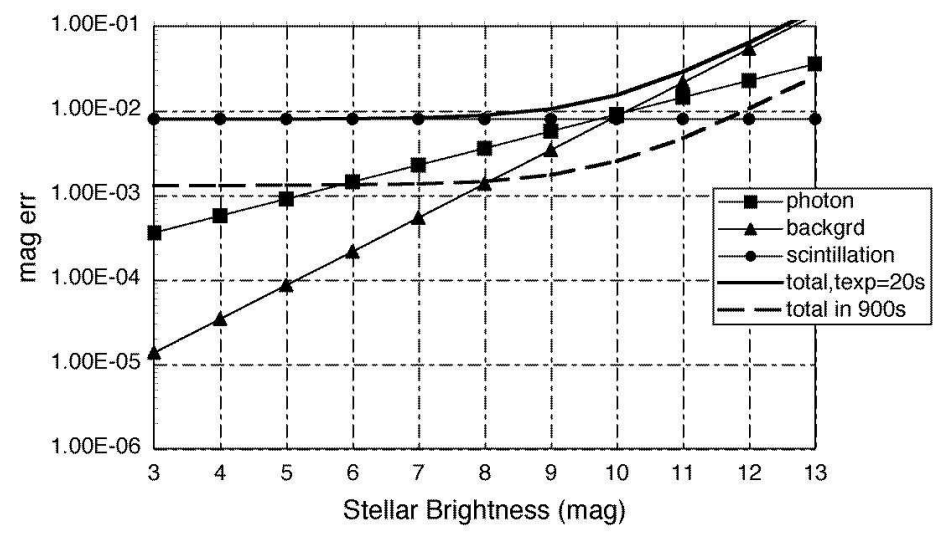

b)

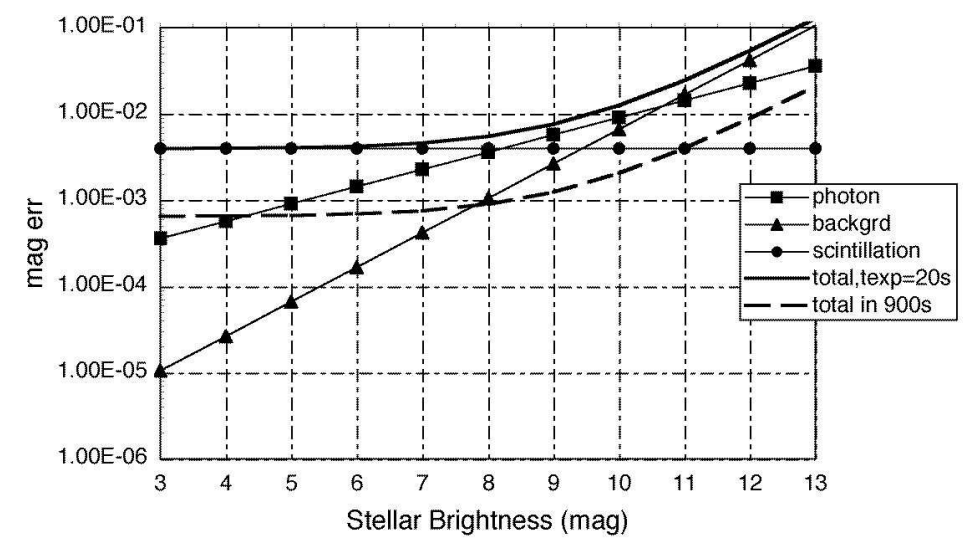

Fig. 2. Noise contributions (as magnitude errors) against stellar magnitude, at a midlatitude site (a) and at Dome C (b). In both cases, 20 second exposures were assumed. The dashed line indicates the summed noise of 20 second exposures during an integration of 900 seconds Further noise sources, such as CCD read noise, dark noise or digitalization noise, are negligible

background dominates the faintest stars. For the conventional site, photon noise from sample stars is a dominant noise in a small range only, around mag 10. For Dome C, stellar photon noise would be dominant in a wider regime from about 8 to 10.5 mag. This would be the consequence of lower scintillation at Dome C, and from a slightly darker night sky.

For the scintillation noise, we assumed half the amplitude of the conventional site (calculated following the equation by Young 1974 for an altitude of $2400 \mathrm{~m}$ 
Title : will be set by the publisher

and an airmass of 1.4), motivated by the excellent scintillation values and large isoplanatic angles recently reported at Dome C (Lawrence et al. 2004). For the photon noise from the sky-background, a value of $21.45 \mathrm{mag} / \operatorname{arcsec}^{2}$ for moonless sky has been assumed for the conventional site (based on values reported in $\mathrm{V}$ for La Palma, Benn \& Ellison 1998), which was changed to $22 \mathrm{mag} / \operatorname{arcsec}^{2}$ for Dome C. It should also be noted, that the very low water content in Dome C's atmosphere will probably avoid the need for the suppression of water-lines, which is contemplated in the current prototype for a mid-latitude site, and for which a filter cutting of wavelengths $>725 \mathrm{~nm}$ is foreseen. Some additional improvements in photon noise (not contemplated in Fig. (2b) at Dome C may therefore be expected. The widening of the magnitude regime dominated by photon noise at Dome $\mathrm{C}$ constitutes a widening of the range of the stellar sample which will be observed by the instruments in an optimized way (meaning that noises cannot be improved upon, except by an enlargement of the optics). The limiting magnitude for a transit search at Dome $\mathrm{C}$ would be about $0.3 \mathrm{mag}$ lower than at a conventional site, which corresponds to about $30 \%$ more sample stars. Another significant gain is expected for the tracking of brighter variable stars, for which precisions better than $1 \mathrm{mmag}$ in 15 minute integrations should be achievable.

The other relevant advantage of Dome $\mathrm{C}$ arises from the much longer yearly observational coverage that is achievable for a large fraction of sample stars. This coverage is calculated in hours per year for a combination of mid-latitude observing sites (Fig. 3a) and for a 'normal' northern site with Dome C (Fig. 3b). For all observing sites, 1500 hours of yearly dark time with good observing conditions have been assumed (a number that is probably very conservative for Dome C). These figures assume that Dome $\mathrm{C}$ allows useful observations of stars as low as $15^{\circ}$ elevation above horizon, whereas for mid-latitude sites, the lowest useful altitude is around $30^{\circ}$ (2 airmasses). Consequently, sample stars south of declinations of $-30^{\circ}$ are circumpolar at Dome $\mathrm{C}$, which allows their observation during the entire dark time. The observational coverage of these stars is typically 2-3 times as long as that of stars only partially visible during a year. Hence, a large fraction of sample stars can achieve in one winter season at Dome $\mathrm{C}$ an observational coverage that corresponds to 2-3 years of observations from a mid-latitude site. One may argue that Dome $\mathrm{C}$ allows coverage only during the winter season. Seasonal coverage is however also the case for the vast majority of stars (those that are not circumpolar) at mid-latitude sites, therefore not making a great difference.

The only moving parts of the current design of PASS are the shutters of the cameras. A placement of that instrument at Dome $\mathrm{C}$ should therefore not present any serious technical challenges. The most reliable way to avoid the effects of the extremely cold winter-time temperatures is probably the enclosure of the entire camera array into an insulated coverage, with plane optical windows in front of each camera. The enclosure would be thermally isolated and its interior kept at a temperature within the specs of the CCD cameras, typically around -20C. Due to the very small plate scale of the CCD cameras (1 pixel corresponds to about 50 arcseconds), any effects onto the seeing from turbulence due to the large temperature difference between inside and outside of the enclosure should be negligible. 
a)

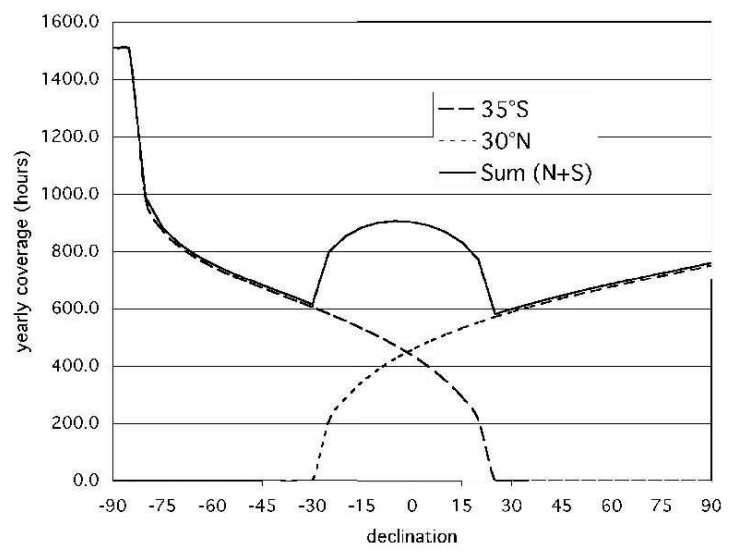

b)

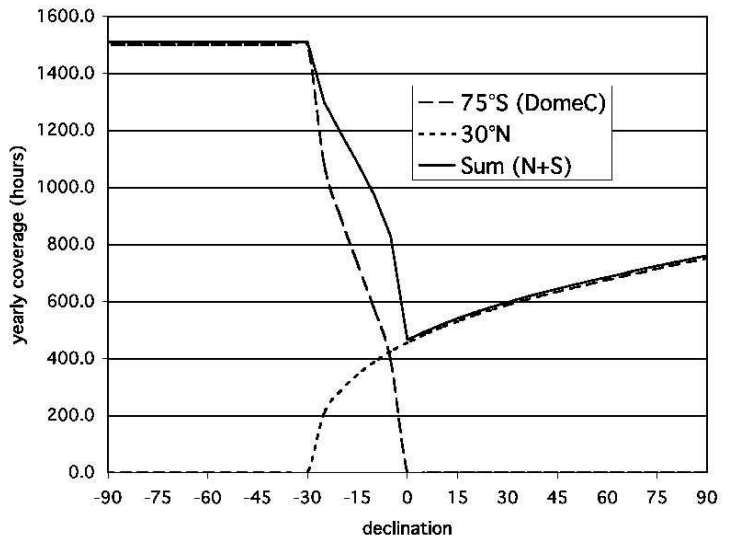

Fig. 3. Yearly observational coverage in dependence on stellar declination, assuming a yearly total of 1500 hours of clear observing conditions. The coverage shown here is the average for stars at any right ascension. a): Coverage for a system consisting of a PASS instrument at a mid-latitude northern and southern site, indicating also the total coverage from both sites (summing coverages of stars that are observable from both sites). b): The southern instrument is now located at Dome C. In this case, stars south of $-30^{\circ}$ are circumpolar, leading to significantly longer coverage. A useful search for short-periodic planetary transits requires at least 600 hours of observing time. Longer times however improve the reliability of detections, and allow the discovery of longer periodic planets.

The only instrumental maintenance required should be an occasional dusting off of snow from the optical windows.

PASS should therefore be a project that is well suited to be positioned at Dome $\mathrm{C}$, due to being able to take advantage of the unique conditions that are offered 
at that location, and due to the simplicity of its instrument. A prototype instrument with one camera is being operated at the Teide observatory, Tenerife, since November 2004. For this instrument, data analysis routines are currently being developed, with the aim to obtain a complete characterization of that instrument and its performance. A placement of this simple and easily transportable system at Dome $\mathrm{C}$ may then be a next step to evaluate the aptness of that location for a later installation of a full PASS array of cameras.

\section{References}

Benn, C. R., \& Ellison, S. L. 1998, La Palma Tech. Note 115, Tech. rep., Isaac Newton Group of Telescopes, La Palma.

Deeg, H., Alonso, R., Belmonte, J., Alsubai, K.,Horne, K. \& Doyle, L. 2004a, PASP, 116, 985.

Deeg, H. J., Alonso, R., Belmonte, J. A., Alsubai, K., Horne, K., Cameron, A.C., Doyle, L.R. 2004b, Astronomische Nachrichten, 325, 643.

Lawrence, J.S., Ashley, M.C.B., Tokovinin, A., Travouilon, T. 2004, Nature, 431, 278.

Young, A. 1974, in Methods of Experimental Physics, Vol. 12, Astrophysics, Part A, ed. N. Carleton (New York: Academic). 